the participants' experience and knowledge about catastrophes.

Methods: The exercise consisted of an interactive board game. The board game represents a map of the ER. Personnel, patients and others were illustrated as game cards. The participants were introduced to a specific situation. The game was played in real time.

Result: The game has been used in regional paediatric educations. The evaluation showed that the game is realistic, educational and has given the participants an increased knowledge and security for potential future catastrophes. The physician class gave the game a 5,8/6 points.

Conclusion: Simulation through interactive role play is considered to be a valuable and educational way of preparing for a catastrophe.

\title{
PO26 - Physical activity - young people's behaviours in Vila Nova de Famalicão
}

\author{
Ilda Fernandes (Portugal) ${ }^{1}$; Manuela Martins (Portugal) ${ }^{1}$; \\ Bárbara Gomes (Portugal)ín Luísa Andrade (Portugal) ${ }^{1}$; \\ Ana Silva (Portugal) ${ }^{1}$
}

${ }^{1} E S E P$

Theme: Physical activity

Keywords: Adolescent, health behaviours, physical exercise, social determinants.

\begin{abstract}
Physical activity in adolescence is of major significance considering body, emotional and behavioural changes. A quantitative, descriptive and transversal study was performed to analyse the physical activity of adolescents from a school community in Vila Nova de Famalicão. A self-administered questionnaire was applied to 1614 adolescents attending secondary and higher education in the academic year 2013/2014, aged between 14-21 years. From total participants, 56.9\% were female and $86.5 \%$ attended secondary school. From the participants engaged in physical activities over the previous week, during 20 minutes, with an increase in sweating and/or in respiratory frequency, 24.9\% referred twice and $12.7 \%$ did not engage in any activities; at least 30 minutes duration, with no visible physiological changes, $10.4 \%$ with daily activities and $41.9 \%$ with no activities and $19.3 \%$ (twice) and $7.6 \%$ (daily) refereed activities to strengthen muscles. Physical activity needs to be promoted to achieve physical and emotional wellbeing among young people.
\end{abstract}

\section{P027 - Road safety - young people's behaviours in Vila Nova de Famalicão}

Ilda Fernandes (Portugal) ${ }^{1}$; Manuela Martins (Portugal) ${ }^{1}$;

Luísa Andrade (Portugal) ${ }^{1}$; Eliana Machado (Portugal) ${ }^{1}$

${ }^{1}$ ESEP

Theme: Accidents 